\title{
Combined Source-Channel Coding of Images under Power and Bandwidth Constraints
}

\author{
Nouman Raja, ${ }^{1}$ Zixiang Xiong, ${ }^{1}$ and Marc Fossorier ${ }^{2}$ \\ ${ }^{1}$ Department of Electrical and Computer Engineering, Texas A\&M University, College Station, TX 77843, USA \\ ${ }^{2}$ Department of Electrical Engineering, University of Hawaii, Honolulu, HI 96822, USA
}

Received 8 June 2006; Revised 9 October 2006; Accepted 14 October 2006

Recommended by Stephen Marshall

This paper proposes a framework for combined source-channel coding for a power and bandwidth constrained noisy channel. The framework is applied to progressive image transmission using constant envelope $M$-ary phase shift key ( $M$-PSK) signaling over an additive white Gaussian noise channel. First, the framework is developed for uncoded $M$-PSK signaling (with $M=2^{k}$ ). Then, it is extended to include coded M-PSK modulation using trellis coded modulation (TCM). An adaptive TCM system is also presented. Simulation results show that, depending on the constellation size, coded $M$-PSK signaling performs 3.1 to $5.2 \mathrm{~dB}$ better than uncoded M-PSK signaling. Finally, the performance of our combined source-channel coding scheme is investigated from the channel capacity point of view. Our framework is further extended to include powerful channel codes like turbo and low-density parity-check (LDPC) codes. With these powerful codes, our proposed scheme performs about one $\mathrm{dB}$ away from the capacity-achieving SNR value of the QPSK channel.

Copyright () 2007 Hindawi Publishing Corporation. All rights reserved.

\section{INTRODUCTION}

Shannon's separation principle [1] states that source coding and channel coding could be optimized individually and then operated in a cascaded system without sacrificing optimality. Therefore, traditionally, channel coders are designed independently of the actual source, while source coders are designed without considering the channel. The resulting coders are then cascaded. However, Shannon's separation principle is valid only for asymptotic conditions such as infinite block length and memoryless channel. Thus, under practical delay and storage constraints, independent designs of source and channel coders are not optimal. This motivates a joint optimal design [2] of the source and channel coders. However, joint optimization is quite complex in practical systems. Not only does the traditional theoretical approach require infinite complexity, but also a completely coupled design seems practically infeasible.

This paper presents a low-complexity technique, which increases the performance of cascaded systems by introducing some amount of coupling between the source coder and the channel coder. Specifically, source- and channel-rate allocations are studied for embedded source coders and a power and bandwidth constrained noisy channel.
The average energy transmitted per source symbol is considered to be an important design parameter when using a power-constrained (e.g., AWGN) channel. Since the transmission rate is the number of bits transmitted per source symbol, if the signal constellation is known, the average energy transmitted per source symbol can be formulated to optimize the end-to-end quantization error of the system. The transmitted bits include source bits and redundant bits. It is therefore important to effectively allocate these bits between the source coder and the channel coder. This allocation is characterized by the choice of a channel code rate. By introducing a bandwidth constraint, this degree of freedom becomes the choices of signal constellation in conjunction with both the channel code rate (resulting in coded modulation) and the source code rate. Thus, there is a tradeoff between modulation, source coding, and channel coding. These components will be examined by jointly optimizing the transmission rate and the channel code rate for a certain class of source and channel codes. Our goal is to minimize the average distortion of a source transmitted over a bandwidth and power constrained noisy channel.

Sherwood and Zeger [3] used a combined source-channel scheme based on Said and Pearlman's set partitioning in hierarchical trees (SPIHT) image-coding algorithm [4]. 
They utilized cyclic redundancy check (CRC) codes [5] and rate-compatible punctured convolutional (RCPC) channel codes for image transmission over binary symmetric channels (BSCs). Since then, a large body of works (see [6] and references therein) has addressed joint source-channel coding (JSCC) for scalable multimedia transmission over both BSCs and packet-erasure channels. Fossorier et al. [7] generalized the scheme of [3] from BSCs to analog binary channels by choosing the average energy per transmitted bit in conjunction with both the source rate and the channel code rate under a power constraint. While the additional degree of freedom makes it possible to achieve higher overall peak signal-to-noise ratio (PSNR) values, it also results in either bandwidth reduction or expansion (with respect to the underlying reference system), the latter being highly undesirable.

The embedded property of SPIHT coded image bitstream has been exploited to provide unequal error protection (UEP) by the use of different channel codes with codes of higher rates allocated to the tail of the bitstream. However, it has been shown in $[8,9]$ that optimal UEP (with much high complexity and longer delay) only offers a small performance gain over optimal equal error protection (EEP) for BSCs. This motivates us to study efficient transmission scheme obtained with constellation expansion, that is, coded modulation, in the spirit of EEP that does not lead to bandwidth expansion as in [7].

Forward error correction is a practical technique for increasing the transmission efficiency of virtually all-digital communication channels. Ungerboeck [10] showed that with TCM, it is possible to achieve asymptotic coding gain of as much as $5.8 \mathrm{~dB}$ in average energy per symbol $\left(E_{s} / N_{0}\right)$ within precisely the same signal spectral bandwidth, by doubling the signal constellation set from $M=2^{k-1}$ to $M=2^{k}$ using a method called set partitioning. The main idea is to maximize Euclidean distance rather than dealing with Hamming distance. The set partitioning strategy maximizes the intrasubset Euclidean distance. It has led to extensive research [11] on finding practical codes and their performance bounds. Viterbi et al. [12] introduced bandwidth-efficient pragmatic codes which generate trellis codes for higher $M$ PSK constellation by using an industry standard rate-1/2 trellis code, at the loss of some performance compared to Ungerboeck codes. Wolf and Zehavi [13] extended pragmatic codes to a wide range of high-rate punctured trellis codes for both PSK and QAM modulations.

This paper proposes a combined source-channel coding framework based on embedded image coders such as SPIHT and JPEG2000. The SNR is chosen in conjunction with the source code rate and the channel code rate under a power constraint. In the meantime, TCM is used in conjunction with a bandwidth constraint. An adaptive TCM system capable of operating at variable rates and modulation formats is designed using punctured TCM codes [14]. Theoretical performance bounds are computed analytically for TCM coding and simulations performed to match the theoretical analysis of TCM coders for our combined source-channel coding system. In addition, simulation results using turbo [15] and
LDPC codes [16] are also presented in this study; the turbo (and LDPC) based source-channel coding system has a gap of 1.2 (and 0.98) dB from the capacity-achieving SNR (SNR gap) value of the QPSK channel.

This paper is organized as follows. In Section 2, we present our combined source-channel coding framework using the SPIHT image coder under power and bandwidth constraints. The SPIHT image coder is reviewed, and both uncoded and coded signaling formats are considered. In Section 3, the proposed framework is applied to $M$-PSK signaling. Theoretical and simulation results for both uncoded and coded cases are presented, followed by the design of an adaptive TCM system. The input constrained capacity for AWGN channels is considered in Section 4. Results from applying both turbo and LPDC codes are also presented. Section 5 concludes the paper.

\section{THE JSCC FRAMEWORK}

\subsection{The SPIHT image coder}

The SPIHT coder by Said and Pearlman [4] is a celebrated wavelet-based embedded image coder. It employs octaveband filter banks for subband/wavelet decomposition of the input image and takes advantage of the fact that the variance of the coefficients decreases from the lowest to the highest bands in the subband pyramid. This SPIHT coding algorithm is an improvement of Shapiro's embedded zerotree wavelet (EZW) coding algorithm [17]. The difference between SPIHT and EZW is that the SPIHT algorithm provides better performance. Both coders outperform JPEG while producing an embedded bitstream, which means that the decoder can stop at any point of the bitstream and still produce a decoded image of commensurate quality. EZW and SPIHT have led to the development of the new JPEG2000 image compression standard. Since both SPIHT and JPEG2000 produce embedded bitstreams, our proposed framework is applicable to both of them. However, we only use the SPIHT image coder in this paper.

\subsection{The proposed framework}

Consider a JSCC system employing the SPIHT image coder emitting bits at rate $r_{s}$, measured in bits per pixel (bpp), where the total number of pixels in the input image(s) is assumed to be $L$. The quality of the decoded image is measured by the mean-squared error (MSE) $D$ as a function of $r_{s}$. Figure 1 depicts the operational distortion-rate function $D\left(r_{s}\right)^{1}$ of SPIHT for the $512 \times 512$ Lena image (with $L=512^{2}$ ), which is monotonically nonincreasing. As the source image is progressively compressed by the SPIHT

\footnotetext{
${ }^{1}$ Since the SPIHT image coder is embedded, $D\left(r_{s}\right)$ can be easily generated by encoding at high rate (e.g., 1 bpp) and decoding at all lower rates. Alternatively, one can use generic models for $D\left(r_{s}\right)$; see [6, Figure 4] SPM for details.
} 


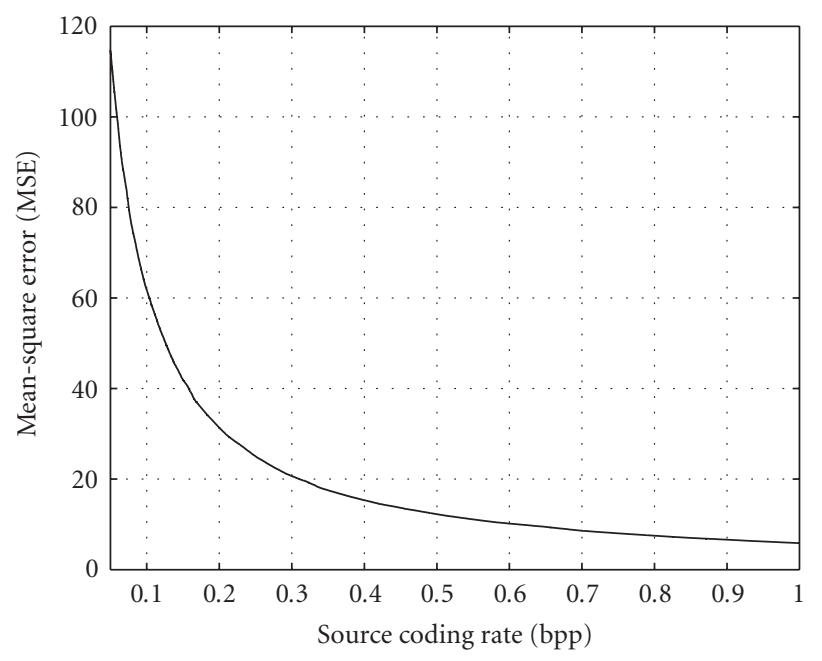

FIGURE 1: Operational distortion-rate function $D\left(r_{s}\right)$ of the SPIHT coder for the $512 \times 512$ Lena image.

coder, decoding stops if a single error occurs. ${ }^{2}$ Thus the average distortion after transmitting an $N$-bit SPIHT bitstream across a channel characterized by its bit-error probability $P_{b}$ can be calculated as

$$
\bar{D}=D\left(\frac{N}{L}\right)\left(1-P_{b}\right)^{N}+\sum_{i=0}^{N-1} D\left(\frac{i}{L}\right)\left(1-P_{b}\right)^{i} P_{b}
$$

If $R$ constellation signals per source sample ${ }^{3}$ are transmitted over the channel using an average energy of $E_{s}$ per transmitted signal, then for a given target power level $P_{0}$ (in maximum permitted energy per source sample), power constrained transmission means $R E_{s} \leq P_{0}$. On the other hand, the bandwidth constraint $R_{0}$ implies a duration per constellation signal (or channel use) of at least $1 / R_{0}$ second, then $R=R_{0}$ implies $E_{s}=P_{0} / R_{0}$ if both the maximum available power and available bandwidth are used.

Let $b_{0}$ be the total number of transmitted symbols for the source image (with $L$ pixels); by the definition of $R$, we have $R=b_{0} / L$. Then $R=R_{0}$ leads to

$$
R=R_{0}=\frac{b_{0}}{L}
$$

In all systems considered in this work, $R_{0}$ is fixed. Equation (2) means $b_{0}$ is a constant in all systems.

If a channel code with rate $r_{c}$ is used for error correction, the maximum number of bits per source sample available for

\footnotetext{
${ }^{2}$ Throughout this paper, we assume that channel errors (if any) can be detected perfectly (e.g., by CRC codes, which are widely used for error detection because of the simplicity of their implementation and the low complexity of both the encoder and the decoder); see, for example, the CRC-RCPC code used in [3].

${ }^{3}$ For transporting images, a source sample corresponds to an image pixel. We use them interchangeably in this paper.
}

source coding is $r_{s}=R_{0} r_{c} k$, with $M=2^{k}$ being the number of modulation levels. Thus, when the maximum available bandwidth is utilized, that is, $R=R_{0}$, we also have

$$
R=\frac{r_{s}}{r_{c} k}
$$

It is assumed that each constellation $\left\{S_{i}\right\}$ used for transmission over an AWGN channel with zero mean and variance $N_{0} / 2$ is associated with a capacity $C_{i}\left(E_{s} / N_{0}\right)$. Shannon's channel coding theorem states that if $r_{c} k<C_{i}\left(E_{s} / N_{0}\right)$, then, $r_{s}$ bits per source sample can be transmitted with an arbitrarily small probability of error and Shannon's separation principle implies that the distortion level $D\left(r_{s}\right)$, corresponding to rate $r_{s}$, can be achieved.

Since $D\left(r_{s}\right)$ is assumed to be a nonincreasing function of $r_{s}$, this simply suggests the selection of the signal constellation that achieves the highest capacity under the power and bandwidth constraints (assuming infinite block lengths).

\subsection{Application to an arbitrary modulation format for an AWGN channel}

We consider the following practical problem based on the embedded SPIHT image coder: for a given AWGN channel with zero mean, variance $N_{0} / 2$, and constraints on both the average power and bandwidth, what is the minimum achievable average MSE of transmitted images, using arbitrary modulation signaling (AMS) for both coded and uncoded systems?

\subsubsection{Uncoded AMS signaling}

The SPIHT image coder is used in conjunction with uncoded $2^{k}$-AMS signaling, that is, $r_{c}=1$. The corresponding average bit-error probability is computed and given as $P_{b}(k)$. For an image (with $L$ pixels) compressed at rate of $r_{s}$ bpp, $r_{c} k b_{0}=$ $k b_{0}=L r_{s}$ source bits are transmitted over the AWGN channel with $b_{0}$ symbols. Due to the embedded nature of the SPIHT coded image bitstream, the average MSE can be expressed as

$$
\begin{aligned}
\bar{D}\left(r_{c}, k\right)= & D\left(r_{s}\right)\left(1-P_{b}(k)\right)^{r_{c} k b_{0}} \\
& +\sum_{i=0}^{r_{c} k b_{0}-1} D\left(\frac{r_{s} i}{r_{c} k b_{0}}\right)\left(1-P_{b}(k)\right)^{i} P_{b}(k),
\end{aligned}
$$

where $D\left(r_{s}\right)$ represents the distortion of the image decoded at rate $r_{s}$ bpp (see Figure 1).

From (2) and (3), the source code rate can be rewritten as $r_{s}=r_{c} k b_{0} / L=k b_{0} / L$, which varies only with $k$ under uncoded signaling. Equation (4) then becomes

$$
\begin{aligned}
\bar{D}(1, k)= & D\left(\frac{k b_{0}}{L}\right)\left(1-P_{b}(k)\right)^{k b_{0}} \\
& +\sum_{i=0}^{k b_{0}-1} D\left(\frac{i}{L}\right)\left(1-P_{b}(k)\right)^{i} P_{b}(k) .
\end{aligned}
$$


Since $D\left(k b_{0} / L\right)$ decreases while $P_{b}(k)$ increases as $k$ increases, it implies that for a given value of $E_{s} / N_{0}$, the optimum choice of $k$ corresponds to the MSE

$$
\bar{D}_{\text {unc,min }}\left(\frac{E_{s}}{N_{0}}\right)=\min _{k} \bar{D}(1, k) .
$$

Intuitively, this choice is justified by the fact that as the channel condition improves (i.e., $E_{s} / N_{0}$ increases), a larger constellation size (i.e., larger value of $k$ ) can be chosen to achieve higher throughput (source) rate $r_{s}$ with lower MSE.

However, a lower average MSE can be obtained if channel coding is combined with the modulation, resulting in coded modulation. The following section illustrates how to do this.

\subsubsection{Coded AMS signaling}

Assume a rate- $r_{c}$ channel code (with $r_{c}<1$ ) is used to transmit images compressed at rate of $r_{s}$ bpp with $2^{k}$-AMS signaling, so that $r_{c} k b_{0}=L r_{s}$. If the corresponding bit-error probability is approximated as $P_{b}(k)$, then the average MSE becomes

$$
\begin{aligned}
\bar{D}\left(r_{c}, k\right)= & D\left(r_{s}\right)\left(1-P_{b}(k)\right)^{r_{c} k b_{0}} \\
& +\sum_{i=0}^{r_{c} k b_{0}-1} D\left(\frac{r_{s} i}{r_{c} k b_{0}}\right)\left(1-P_{b}(k)\right)^{i} P_{b}(k) \\
= & D\left(\frac{r_{c} k b_{0}}{L}\right)\left(1-P_{b}(k)\right)^{r_{c} k b_{0}} \\
& +\sum_{i=0}^{r_{c} k b_{0}-1} D\left(\frac{i}{L}\right)\left(1-P_{b}(k)\right)^{i} P_{b}(k) .
\end{aligned}
$$

We optimize (7) over $r_{c}$ and $k$ for fixed $b_{0}$ and $L$ to obtain

$$
\bar{D}_{\text {cod, } \min }\left(\frac{E_{s}}{N_{0}}\right)=\min _{r_{c}, k} \bar{D}\left(r_{c}, k\right) .
$$

In terms of the PSNR in $\mathrm{dB}$, it becomes

$$
\operatorname{PSNR}=10 \log _{10} \frac{255^{2}}{\bar{D}_{\mathrm{opt}}\left(E_{s} / N_{0}\right)}
$$

where $\bar{D}_{\text {opt }}\left(E_{s} / N_{0}\right)$ is chosen as (6) or $(8)$.

Depending on the channel condition, we optimize both the channel code rate and modulation format for minimum distortion (or maximum PSNR).

\section{APPLICATION OF THE JSCC FRAMEWORK TO $M$ - ARY PSK MODULATION}

\subsection{Phase shift keying (PSK)}

PSK is a combined energy modulation scheme in which the source information is contained in the phase of the transmitted carrier. For a given value of $E_{s} / N_{0}$, the bit-error probability $P_{b}(k)$ of $M$-PSK signaling over an AWGN channel using

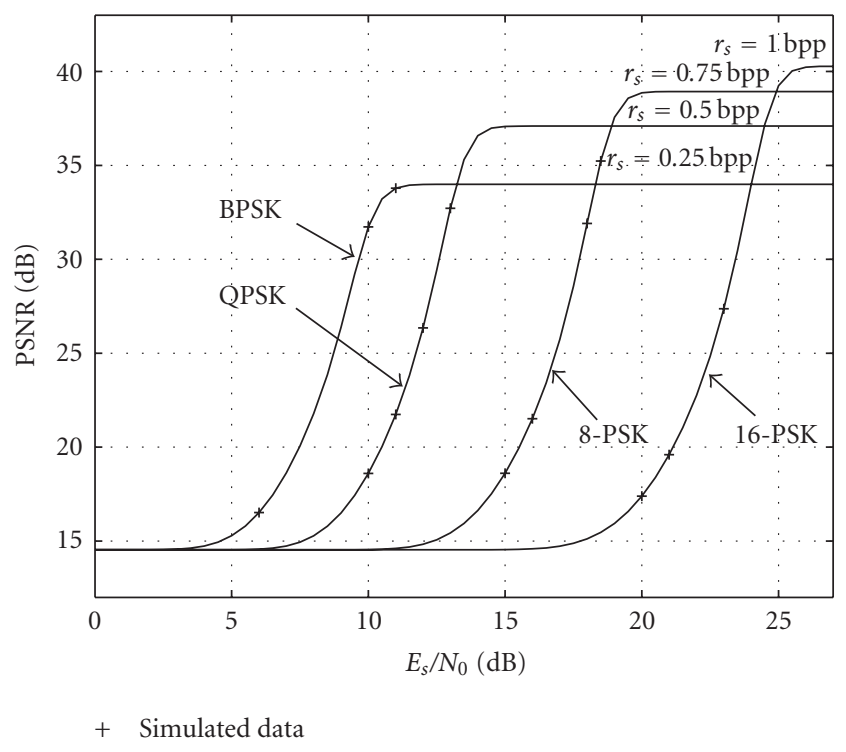

FIGURE 2: PSNR versus $E_{s} / N_{0}$ performance of using an uncoded $M$ PSK system (with $M=2^{k}$ for $\left.k=1,2,3,4\right)$ for transmitting the SPIHT compressed $512 \times 512$ Lena image using $b_{0}=65,536$ symbols. The source coding rate is $0.25,0.5,0.75$, and $1 \mathrm{bpp}$, respectively, for $k=1,2,3$, and 4 .

gray mapping can be approximated as [18]

$$
P_{b}(k) \approx 2 Q\left(\sqrt{2 E_{s} / N_{0}} \sin \frac{\pi}{M}\right)
$$

Figure 2 depicts the performance of the JSCC scheme by transmitting the $512 \times 512$ Lena image using uncoded $M$ PSK signaling (with $M=2^{k}$ for $k=1,2,3,4$ ). Both simulated results " $(+)$ " and the corresponding theoretical values are shown. The bandwidth and power constraints are satisfied by fixing the number of constant energy PSK symbols to $b_{0}=65,536$, meaning $R_{0}=0.25$ and $r_{s}=0.25,0.5,0.75$, and $1 \mathrm{bpp}$, respectively, for $k=1,2,3$, and 4 . For each fixed $k$, as $E_{s} / N_{0}$ increases from $0 \mathrm{~dB}, P_{b}(k)$ decreases, and the system's PSNR performance improves until it reaches its ceiling when $P_{b}(k)=0$ and $\bar{D}(1, k)=D\left(r_{s}\right)$, means the ceiling point is determined by SPIHT's source coding performance at rate $r_{s}$. Using different $k$ 's, there is no performance difference ${ }^{4}$ at very low $E_{s} / N_{0}$ ( since $P_{b}(k) \approx 1$ for all $k$ ), however, since $r_{s}$ is higher for larger $k$, the system performance plateaus sooner at lower PSNR with smaller $k$ than with larger $k$. The best system performance corresponds to the envelop of the different PSNR versus $E_{s} / N_{0}$ curves.

The uncoded system performs poorly at low $E_{s} / N_{0}$. To improve this performance, coded modulation techniques like TCM should be used.

\footnotetext{
4 The $14.53 \mathrm{~dB}$ minimum PSNR corresponds to using the default decoded image with constant pixel value 128 for Lena. We note that the image quality should be at least $30 \mathrm{~dB}$ in PSNR to have no noticeable visual artifacts. By starting at the minimum $14.5 \mathrm{~dB}$, we intend to provide the whole picture of results that are verified by simulations.
} 


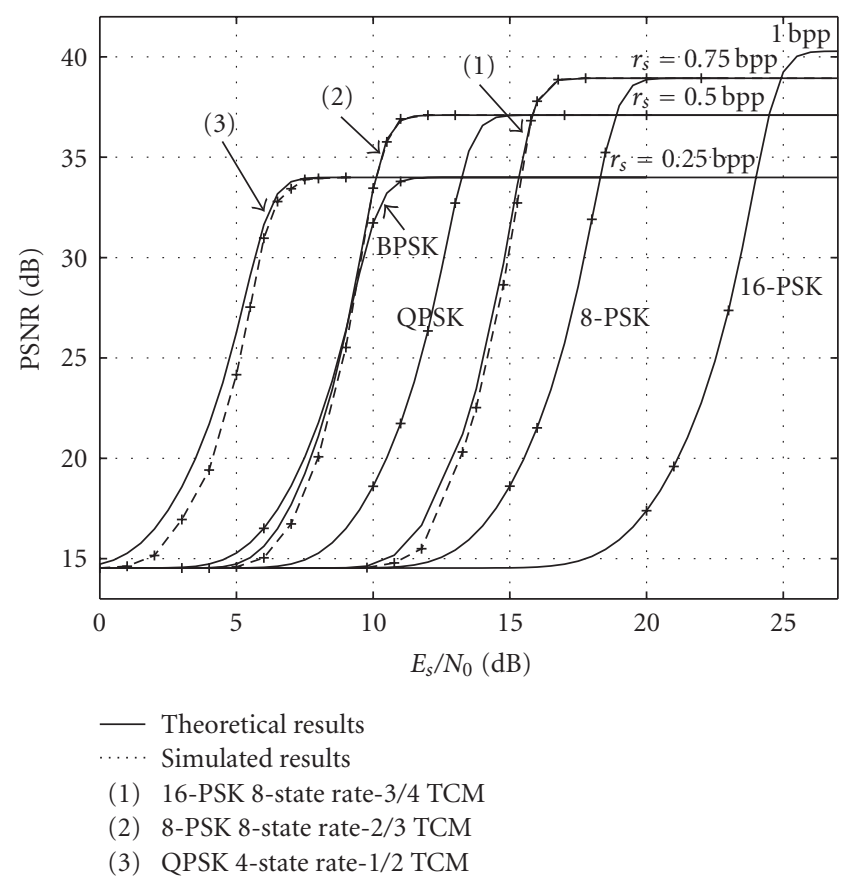

FIgURE 3: PSNR versus $E_{s} / N_{0}$ performance of using a TCM system for transmitting the SPIHT compressed $512 \times 512$ Lena image using 65,536 symbols. The source coding rate for QPSK 4-state rate1/2 TCM, 8-PSK 8-state rate-2/3 TCM, and 16-PSK 8-state rate-3/4 TCM is $0.25,0.5$, and $0.75 \mathrm{bpp}$, respectively. Both theoretical curve based on (11) and respective simulation results are provided. The performance of uncoded systems of Figure 2 is also included for comparison purposes.

\subsection{Trellis-coded modulation (TCM)}

TCM codes [10] introduce the redundancy required for error control without increasing the signal bandwidth by expanding the signal constellation size. Now, symbol mapping becomes part of the TCM code design and it is done in a special way called set partitioning. Ungerboeck [10] showed that it is possible to achieve an asymptotic coding gain of as much as $5.8 \mathrm{~dB}$ in $E_{s} / N_{0}$ without any bandwidth expansion. The probability of symbol error for transmission over noisy channels is a function of the minimum Euclidean distance $d_{\text {free }}$ between pairs of distinct signal sequences. If $b_{d \text { free }}$ is the total number of information bit errors associated with the erroneous paths at distance $d_{\text {free }}$ from the transmitted one, averaged over all possible transmitted paths, we have a probability of bit error [19] of

$$
P_{b}(k) \approx \frac{b_{\text {dree }}}{r_{c}} Q\left(\sqrt{\frac{d_{\text {free }}^{2} E_{s}}{2 N_{0}}}\right) .
$$

at sufficiently high $E_{s} / N_{0}$.

Figure 3 depicts the performance of three coded systems that uses 4-state rate-1/2 TCM (with QPSK), 8-state rate2/3 TCM (with 8-PSK), and 8-state rate-3/4 TCM (with 16PSK), respectively, again for transmitting the $512 \times 512$ Lena image using 65,536 symbols ( or $R_{0}=0.25$ ). The corresponding source coding rate $r_{s}=R_{0} r_{c} k$ is $0.25,0.5$, and $0.75 \mathrm{bpp}$,
TABLE 1: The best choice of channel code rate and signal constellation (and their associated source coding rate) corresponding to different $E_{s} / N_{0}$ ranges based on Figure 4 for our adaptive TCM system when transmitting the SPIHT compressed $512 \times 512$ Lena image using 65,536 symbols.

\begin{tabular}{l|c|c|c}
\hline $\begin{array}{l}E_{s} / N_{0} \\
\text { range }(\mathrm{dB})\end{array}$ & $\begin{array}{l}\text { Channel } \\
\text { code rate } r_{c}\end{array}$ & $\begin{array}{l}\text { Signal } \\
\text { constellation }\end{array}$ & $\begin{array}{l}\text { Source coding } \\
\text { rate } r_{s}(\mathrm{bpp})\end{array}$ \\
\hline $0.00-6.91$ & $1 / 2$ & QPSK & 0.25 \\
$6.91-7.48$ & $2 / 3$ & QPSK & 0.33 \\
$7.48-10.8$ & $3 / 4$ & QPSK & 0.375 \\
$10.8-12.45$ & $2 / 3$ & 8-PSK & 0.5 \\
$12.45-15.6$ & $5 / 6$ & 8-PSK & 0.625 \\
$15.60-25.00$ & $3 / 4$ & 16-PSK & 0.75 \\
\hline
\end{tabular}

respectively. Both theoretical curve based on (11) and respective simulation results are provided. It is seen that there exists a mismatch between the theoretical and simulation values at low $E_{s} / N_{0}$. This is because the BER in (11) is approximated using only the error paths at distance $d_{\text {free }}$.

The performance of uncoded systems of Figure 2 are also included for comparison purposes. It is seen that, at the same $r_{s}$, a TCM coded system performs better than an uncoded system at low $E_{s} / N_{0}$.

We note that a similar approach has been presented in [20] for robust video coding. However in [20], binary channel coding with gray-mapped QPSK signaling is considered in conjunction with an enhancement, which allows one to select two rotated versions of the QPSK constellation, resulting in nonuniform 8-PSK signaling. Contrary to our proposed scheme, channel coding in [20] is realized independently of the modulation so that independent parallel binary channels are considered at the receiver.

\subsection{Adaptive TCM system}

The performance of the TCM system depicted in Figure 3 still saturates quickly and in some regions of $E_{s} / N_{0}$ values, the uncoded system performs better. Moreover, each configuration requires a separate code. Hence for practical use with variable channel conditions, the JSCC-TCM system presented above is not suitable. We thus devise a single encoder-decoder TCM system based on punctured codes [14]. It is assumed that the transmitter is able to perform adaptive modulation, which can be achieved, for example, with the help of channel side information.

Figure 4 presents the performance of this adaptive TCM system. It employs a single 64 -state rate- $1 / 2$ TCM code in [12] as its base code, which has reasonable decoding complexity. By varying the puncturing rate (which leads to different $r_{c}$ 's) and $k$ (or the constellation size $M$ ), a number of system configurations are generated and their performance presented. The best performance of this adaptive TCM system is the envelop of all PSNR versus $E_{s} / N_{0}$ curves. Table 1 summarizes the best choices of $r_{c}$ and constellation size $M=2^{k}$ with PSK (and the associated $r_{s}=R_{0} r_{c} k$ ) corresponding to different $E_{s} / N_{0}$ ranges. 


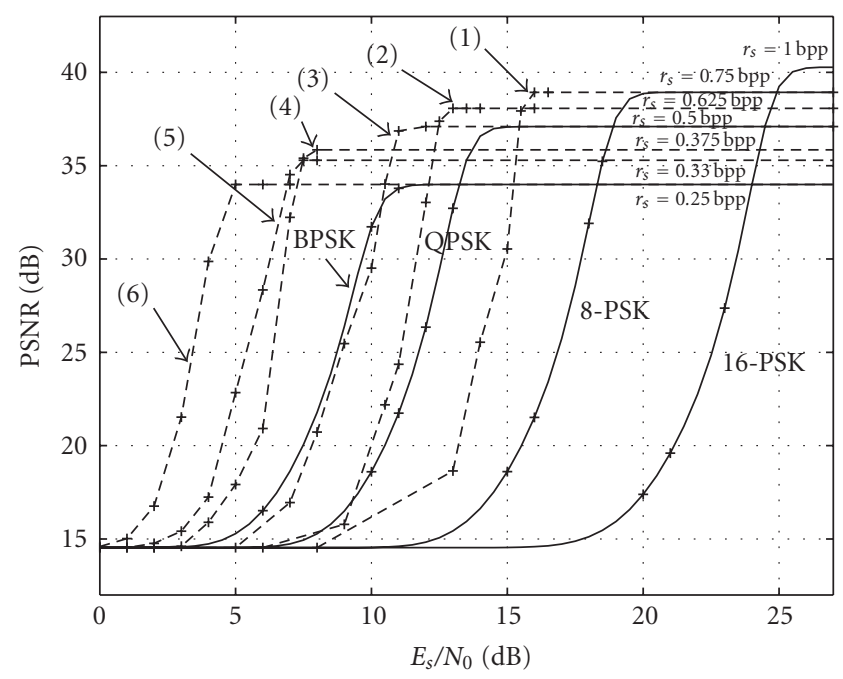

(1) 16-PSK 64-state rate-1/2 punc $3 / 4$ TCM

(2) 8-PSK 64 -state rate- $1 / 2$ punc $5 / 6 \mathrm{TCM}$

(3) 8-PSK 64 -state rate- $1 / 2$ punc $2 / 3$ TCM

(4) QPSK 64-state punc rate-3/4 TCM

(5) QPSK 64-state punc rate-2/3 TCM

(6) QPSK 64-state rate-1/2 TCM

FIGURE 4: PSNR versus $E_{s} / N_{0}$ performance of our adaptive TCM system for transmitting the SPIHT compressed $512 \times 512$ Lena image using 65,536 symbols. Numbers next to the performance ceilings are the source coding rates $r_{s}=R_{0} r_{c} k$, with $R_{0}=0.25$ and $M=2^{k}$ being the constellation size.

It is seen from Figure 4 that our QPSK 64-state rate$1 / 2$ TCM coded system performs $5.2 \mathrm{~dB}$ better than uncoded BPSK signaling, and that our 8-PSK 64-state rate-2/3 TCM coded system and 16-PSK 64-state rate-3/4 TCM coded system performs $3.1 \mathrm{~dB}$ better than uncoded QPSK and 8-PSK signaling, respectively.

So far, the performance of our TCM-based JSSC scheme is studied in terms of $E_{s} / N_{0}$. In the next section, the performance is studied from a channel capacity perspective using powerful channel codes.

\section{PERFORMANCE OF JSCC USING CAPACITY-APPROACHING CODES}

\subsection{Channel capacity}

The capacity of a discrete input continuous output memoryless (e.g., AWGN) channel is given as

$$
C_{M}=\max _{p\left(\mathbf{x}_{m}\right)} \sum_{m=1}^{M} \int_{-\infty}^{\infty} p\left(\mathbf{x}_{m}, \mathbf{y}\right) \log _{2} \frac{p\left(\mathbf{y} \mid \mathbf{x}_{m}\right)}{p(\mathbf{y})} d \mathbf{y}
$$

If $b_{0}$ symbols are transmitted over this channel, then the minimum achievable distortion is given by $D\left(b_{0} C_{M} / L\right)$,

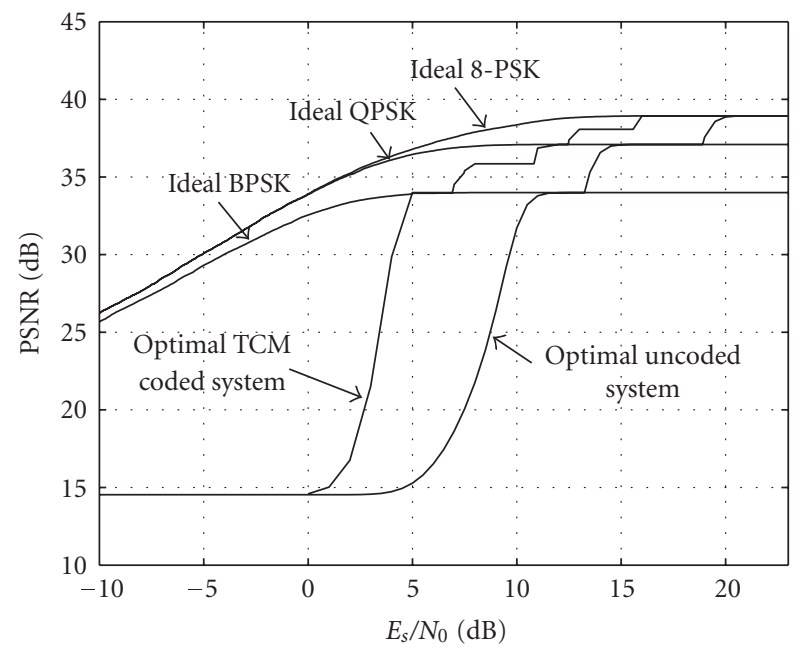

FIGURe 5: The best PSNR versus capacity-achieving $E_{s} / N_{0}$ performance of using our JSCC system for transmitting the SPIHT compressed $512 \times 512$ Lena image using 65,536 symbols.

where $D(\cdot)$ is the operational distortion-rate function (see Figure 1) of the SPIHT image coder.

In Figure 5, the performance of the JSCC framework, employing the adaptive TCM system (see Section 3.3) and uncoded $M$-PSK modulation, is compared with the minimum achievable distortion. We observe that there still remain large SNR gaps at the low SNR range. The performance can be improved by employing capacity-approaching random codes like turbo [15] and LDPC codes [16] for low $E_{s} / N_{0}$ values (although theoretical expressions are no longer feasible).

\subsection{Turbo-coded JSCC system}

A turbo encoder consists of two binary rate- $1 / 2$ recursive systematic convolutional (RSC) encoders separated by an interleaver. Unfortunately, the presence of an interleaver complicates the structure of a turbo code trellis, and a decoder based on maximum-likelihood estimation cannot be used. Thus a suboptimal iterative decoder based on the a posteriori probability (APP) binary BCJR [21] algorithm is used. Given the channel output sequence, the BCJR decoder estimates the bit probability.

In the case of turbo coded modulation, there are a couple of techniques that can be used. A turbo system can be designed specifically for the corresponding modulation scheme $[22,23]$. For example, a symbol interleaver is used in [23] and a symbol-based BCJR algorithm is replaced at the decoder side. The technique in [24] uses a direct extension of binary turbo codes. The output of the binary turbo encoder is gray mapped to some constellation symbols. The received symbols are demodulated and the log-likelihood ratio (LLR) of each bit in the symbol is computed. This soft information is then passed to the decoder. This scheme is simple and easily extendable. We designed turbo codes of rate-1/2 with 16 state QPSK and rates $1 / 3$ and $2 / 3$ with 16 -state 8 -PSK using 


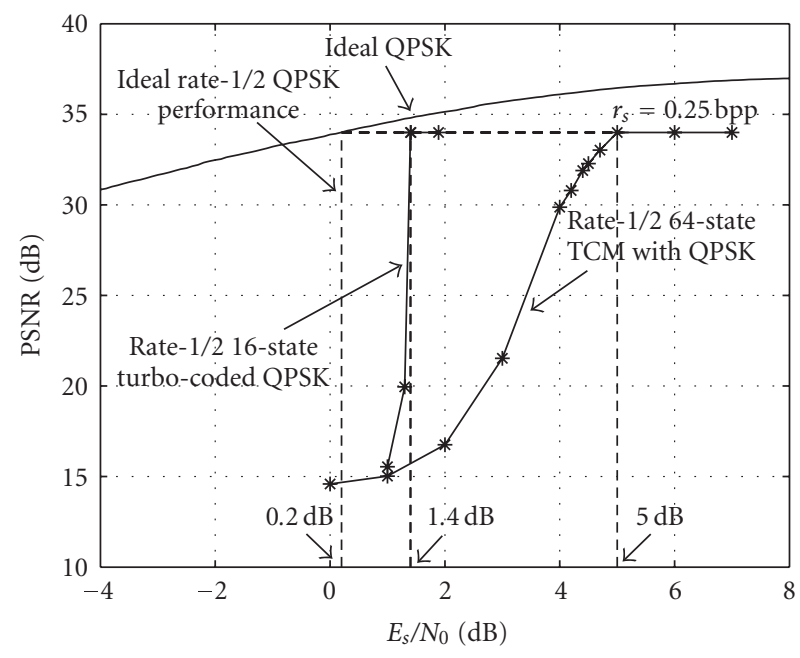

FIGURE 6: PSNR versus $E_{s} / N_{0}$ performance of using rate-1/2 turbo coded QPSK for transmitting the SPIHT compressed $512 \times 512$ Lena image using 65,536 symbols (with $r_{s}=0.25 \mathrm{bpp}$ ).

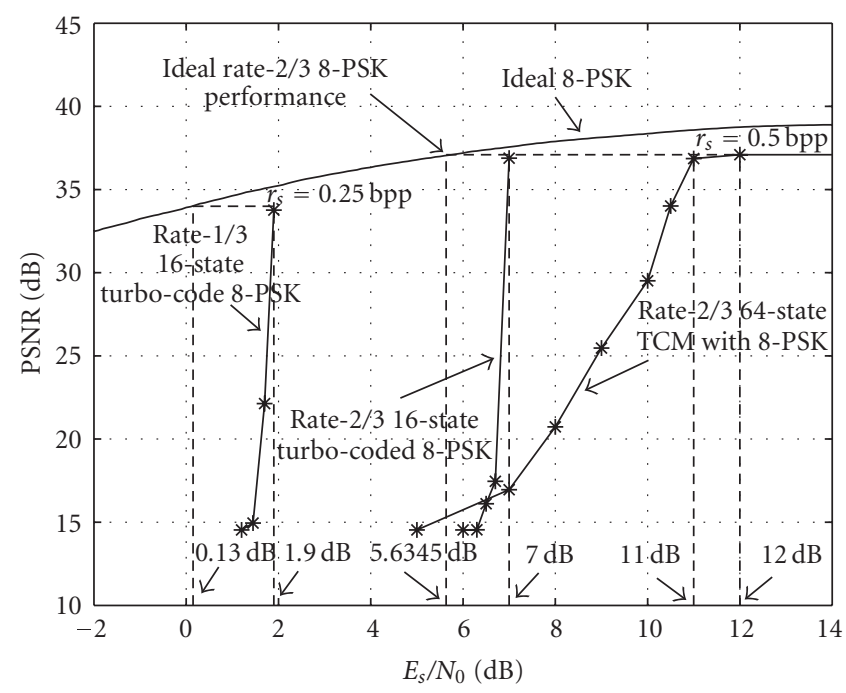

FIgURE 7: PSNR versus $E_{s} / N_{0}$ performance of using rates $1 / 3$ and $2 / 3$ turbo coded 8-PSK for transmitting the SPIHT compressed $512 \times$ 512 Lena image using 65,536 symbols. The corresponding source coding rate is 0.25 and $0.5 \mathrm{bpp}$, respectively.

this technique. The corresponding performances using an Srandom interleaver with a block size of 6,096 are shown in Figures 6 and 7, respectively.

In our simulations, we transmitted 11 blocks, meaning $11 \times 6096=67,056$ symbols, and the reported performance of turbo codes is calculated based on considering the first 65,536 symbols only. It is seen from Figure 6 that the rate-1/2 turbo code is $1.4-0.2=1.2 \mathrm{~dB}$ away from the capacity for QPSK; and turbo codes with coded modulation can achieve an additional gain of $3.6 \mathrm{~dB}$ over their TCM code counterpart. Figure 7 indicates that the rate- $1 / 3$ and rate- $2 / 3$ turbo

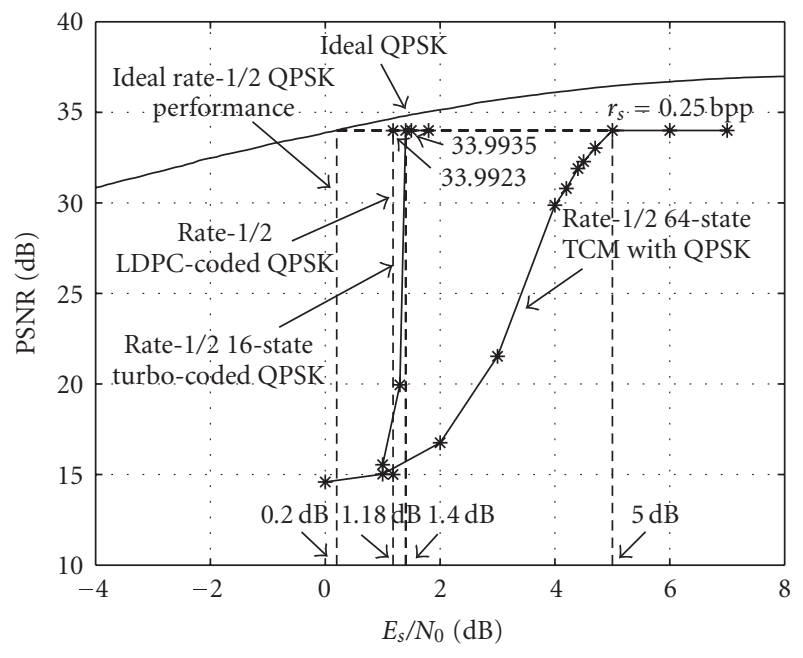

FIGURE 8: PSNR versus $E_{s} / N_{0}$ performance of using a rate- $1 / 2$ LDPC-coded QPSK for transmitting the SPIHT compressed $512 \times$ 512 Lena image using 65,536 symbols (with $r_{s}=r_{s}=0.25 \mathrm{bpp}$ ).

codes are $1.9-0.13=1.77$ and $7-5.6345=1.3655 \mathrm{~dB}$ away from capacity for 8-PSK, respectively. The performance for our turbo coded system degrades at low SNR because of increased noise power.

The above turbo codes are on average $1.4 \mathrm{~dB}$ away from near-Shannon-limit error-correction performance. This gap can be further reduced by increasing the frame size but at the cost of increased computation and latency, and/or by using other types of turbo codes designed specifically for coded modulation. An alternate is to use low-complexity LDPC codes.

\subsection{LDPC-coded JSCC system}

An LDPC code is completely specified by its parity check matrix. Extensive research works (e.g., [25]) have been conducted on the design of LDPC codes. When designed carefully, irregular LDPC codes can perform very closely to the capacity of typical channels.

As for the case of turbo coded modulation, similar techniques have been developed for LDPC codes $[26,27]$. We have designed a binary LDPC code of length $2 \times 65,536$ bits for QPSK signaling using the approach of [26] (with edge profiles $\lambda(x)=0.4717 x+0.33358 x^{2}+0.0108 x^{3}+0.04257 x^{4}+$ $0.007025 x^{7}+0.004925 x^{9}+0.12996 x^{11}$ and $\rho(x)=0.28125 x^{6}+$ $\left.0.70942 x^{7}+0.00934 x^{8}\right)$ and applied to our combined sourcechannel coding system. The results are shown in Figure 8. In our experiments, we set the maximum number of LDPC decoding iterations to be 60 (between the demodulator and the LDPC decoder) and 25 (for the LDPC decoder). Because there is always a probability of decoding error, we run the same image transmission 5,000 times at the operating $E_{s} / N_{0}$ and make sure that correct image decoding is guaranteed at least 996 out of every 1,000 runs before reporting the averaged PSNR results. This makes sure that the effect on the PSNR performance due to the probability of error is 
TABLE 2: Gains achieved with channel coding techniques (using rate-1/2 code and QPSK signaling) when transmitting the SPIHT compressed $512 \times 512$ Lena image using 65,536 symbols. The source coding rate is $r_{s}=0.25 \mathrm{bpp}$.

\begin{tabular}{l|cc}
\hline $\begin{array}{l}\text { Modulation } \\
\text { scheme }\end{array}$ & $\begin{array}{l}\text { Gain over uncoded } \\
\text { system }(\mathrm{dB})\end{array}$ & SNR gap $(\mathrm{dB})$ \\
\hline Trellis coded & 5.2 & 4.8 \\
Turbo coded & 8.8 & 1.2 \\
LDPC coded & 9.02 & 0.98 \\
\hline
\end{tabular}

negligible at the operating $E_{s} / N_{0}$. Figure 8 indicates that the average decrease in image quality due to LDPC decoding errors is $33.9935-33.9923=0.0012 \mathrm{~dB}$ in PSNR (because all four errors in every 1,000 runs in our experiments occur towards the end of the source bitstream). It is also seen that our JSCC system with LDPC codes (operating at $E_{s} / N_{0}=1.18 \mathrm{~dB}$ ) is $0.98 \mathrm{~dB}$ away from the capacity and it performs $0.22 \mathrm{~dB}$ and $3.82 \mathrm{~dB}$ better than the turbo system and TCM system, respectively, for the QPSK system.

The overall performance achieved by our scheme with rate- $1 / 2$ code using various coding schemes (e.g., TCM, turbo and LDPC codes) for QPSK modulation is summarized in Table 2. Similar results can be achieved by using turbo and LDPC codes with various rates and M-PSK modulations.

\section{CONCLUSIONS}

In this paper, a general framework for determining the optimal source-channel coding tradeoff for a power and bandwidth constrained channel has been presented. It addresses a potential shortcoming of [7] with respect to bandwidth expansion. It also offers an additional degree of freedom with respect to the EEP/UEP approaches of $[3,8,9]$, as well as a means of improvement. This framework has been applied to progressive image transmission with constant envelope $M$ PSK TCM signaling over the AWGN channel. An adaptive $M$ PSK TCM system employing a single encoder-decoder pair is also presented. Our combined source-channel coding approach is close to be optimal, when used in conjunction with strong random coding techniques. Extensions to other signaling constellations or channel models follow in a straightforward manner. A particularly well-suited example for PAM signaling over a fading channel is the JSCC scheme proposed in [28] in which several PAM constellations can be chosen adaptively.

\section{REFERENCES}

[1] C. Shannon, "A mathematical theory of communication," The Bell System Technical Journal, vol. 27, pp. 379-423, 1948.

[2] B. Hochwald and K. Zeger, "Tradeoff between source and channel coding," IEEE Transactions on Information Theory, vol. 43, no. 5, pp. 1412-1424, 1997.
[3] G. Sherwood and K. Zeger, "Progressive image coding for noisy channels," IEEE Signal Processing Letters, vol. 4, no. 7, pp. 189-191, 1997.

[4] A. Said and W. Pearlman, "A new, fast, and efficient image codec based on set partitioning in hierarchical trees," IEEE Transactions on Circuits and Systems for Video Technology, vol. 3, no. 3, pp. 243-250, 1996.

[5] T. Ramabadran and S. Gaitonde, "A tutorial on CRC computations," IEEE Micro, vol. 8, no. 4, pp. 62-75, 1988.

[6] R. Hamzaoui, V. Stankovic, and Z. Xiong, "Optimized error protection of scalable image bitstreams," IEEE Signal Processing Magazine, vol. 22, no. 6, pp. 91-107, 2005.

[7] M. Fossorier, Z. Xiong, and K. Zeger, "Progressive source coding for a power constrained Gaussian channel," IEEE Transactions on Communications, vol. 49, no. 8, pp. 1301-1306, 2001.

[8] V. Chande and N. Farvardin, "Progressive transmission of images over memoryless noisy channels," IEEE Journal on Selected Areas in Communications, vol. 18, no. 6, pp. 850-860, 2000.

[9] M. Farshchian, S. Cho, and W. Pearlman, "Optimal error protection for real-time image and video transmission," IEEE Signal Processing Letters, vol. 11, no. 10, pp. 780-783, 2004.

[10] G. Ungerboeck, "Channel coding with multilevel/phase signals," IEEE Transactions on Information Theory, vol. 28, no. 1, pp. 55-67, 1982.

[11] E. Biglieri, D. Divsalar, P. McLane, and M. Simon, Introduction to Trellis-Coded Modulation with Applications, Macmillan, New York, NY, USA, 1991.

[12] A. Viterbi, J. Wolf, E. Zehavi, and R. Padovani, "A pragmatic approach to trellis-coded modulation," IEEE Communications Magazine, vol. 27, no. 7, pp. 11-99, 1989.

[13] J. Wolf and E. Zehavi, " $P^{2}$ codes: pragmatic trellis codes utilizing punctured convolutional codes," IEEE Communications Magazine, vol. 33, no. 2, pp. 94-99, 1995.

[14] J. Kim and G. Pottie, "On punctured trellis-coded modulation," IEEE Transactions on Information Theory, vol. 42, no. 2, pp. 627-636, 1996.

[15] C. Berrou and A. Glavieux, "Near optimum error correcting coding and decoding: turbo-codes," IEEE Transactions on Communications, vol. 44, no. 10, pp. 1261-1271, 1996.

[16] R. Gallager, Low-Density Parity-Check Codes, MIT Press, Cambridge, Mass, USA, 1963.

[17] J. Shapiro, "Embedded image coding using zerotrees of wavelet coefficients," IEEE Transactions on Signal Processing, vol. 41, no. 12, pp. 3445-3462, 1993.

[18] J. Proakis, Digital Communications, McGraw-Hill, New York, NY, USA, 4th edition, 2001.

[19] S. Wicker, Error Control Coding Systems for Digital Communication and Storage, Prentice-Hall, Englewood Cliffs, NJ, USA, 1995.

[20] Y. Pei and J. Modestino, "Multi-layered video transmission over wireless channels using an adaptive modulation and coding scheme," in Proceedings of International Conference on Image Processing (ICIP '01), pp. 1009-1012, Thessaloniki, Greece, October 2001.

[21] L. Bahl, J. Cocke, F. Jelinek, and J. Raviv, "Optimal decoding of linear codes for minimizing symbol error rate," IEEE Transactions on Information Theory, vol. 20, no. 2, pp. 284-287, 1974.

[22] C. Fragouli and R. Wesel, "Turbo-encoder design for symbolinterleaved parallel concatenated trellis-coded modulation," IEEE Transactions on Communications, vol. 49, no. 3, pp. 425435, 2001. 
[23] P. Robertson and T. Worz, "Bandwidth-efficient turbo trelliscoded modulation using punctured component codes," IEEE Journals on Selected Areas in Communications, vol. 16, no. 2, pp. 206-218, 1998.

[24] S. Goff, A. Glavieux, and C. Berrou, "Turbo-codes and high spectral efficiency modulation," in Proceedings of International Conference on Creationism (ICC '94), vol. 3, pp. 1255-1259, New Orleans, La, USA, May 1994.

[25] T. Richardson, A. Shokrollahi, and R. Urbanke, "Design of capacity-approaching irregular low-density parity-check codes," IEEE Transactions on Information Theory, vol. 47, no. 1, pp. 619-637, 2001.

[26] K. Narayanan, I. Altunbas, and R. Narayanaswami, "Design of codes for minimum shift keying based on density evolution," IEEE Transactions on Communications, vol. 51, no. 8, pp. 1283 1295,2003

[27] J. Hou, P. H. Siegel, L. Milstein, and H. Pfister, "Capacityapproaching bandwidth-efficient coded modulation schemes based on low-density parity-check codes," IEEE Transactions on Information Theory, vol. 49, no. 9, pp. 2141-2154, 2003.

[28] M. Alouini, X. Tang, and A. Goldsmith, "An adaptive modulation scheme for simultaneous voice and datatransmission over fading channels," IEEE Journal on Selected Areas in Communications, vol. 17, no. 5, pp. 837-850, 1999.

Nouman Raja received the B.S degree in electronics engineering from G.I.K. Institute of Engineering Sciences and Technology, Pakistan, and M.S. degree in electrical engineering from Texas A\&M University, College Station, Texas, in 2001 and 2003, respectively. He joined Mid-American Equipment Company, Chicago, in 2004, where as a Project Engineer he has been working on designing customized motion control equipment.

Zixiang Xiong received the Ph.D. degree in electrical engineering in 1996 from the University of Illinois at Urbana-Champaign. From 1995 to 1997, he was with Princeton University, first as a Visiting Student, then as a Research Associate. From 1997 to 1999, he was with the University of Hawaii. Since 1999, he has been with the Department of Electrical and Computer Engineering at Texas A\&M University, where he is an

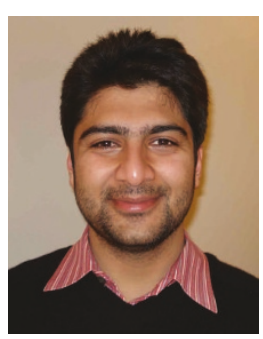
Associate Professor. He spent the summers of 1998 and 1999 at Microsoft Research, Redmond, Washington. He is also a Regular Visitor to Microsoft Research in Beijing. He received a National Science Foundation Career Award in 1999, an Army Research Office Young Investigator Award in 2000, and an Office of Naval Research Young Investigator Award in 2001. He also received Faculty Fellow Awards in 2001, 2002, and 2003 from Texas A\&M University. He served as Associate Editor for the IEEE Transactions on Circuits and Systems for Video Technology (1999-2005), the IEEE Transactions on Image Processing (2002-2005), and the IEEE Transactions on Signal Processing (2002-2006). He is currently an Associate Editor for the IEEE Transactions on Systems, Man, and Cybernetics (part B) and a Member of the multimedia signal processing technical committee of the IEEE Signal Processing Society. He is the Publications Chair of GENSIPS'06 and ICASSP'07 and the Technical Program Committee Cochair of ITW'07.
Marc Fossorier received the B.E. degree from the National Institute of Applied Sciences (INSA.), Lyon, France, in 1987, and the M.S. and Ph.D. degrees from the University of Hawaii at Manoa, Honolulu, USA, in 1991 and 1994, respectively, all in electrical engineering. In 1996, he joined the Faculty of the University of Hawaii, Honolulu, as an Assistant Professor of electrical engineering. He was promoted to Associate Professor in 1999 and to Professor in 2004. His research interests include decoding techniques for linear codes, communication algorithms, and statistics. He is a recipient of a 1998 NSF Career Development Award and became IEEE Fellow in 2006. He has served as Editor for the IEEE Transactions on Information Theory since 2003, as Editor for the IEEE Communications Letters since 1999, as Editor for the IEEE Transactions on Communications from 1996 to 2003, and as Treasurer of the IEEE Information Theory Society from 1999 to 2003. Since 2002, he has also been an Elected Member of the Board of Governors of the IEEE Information Theory Society which he is currently serving as Second Vice-President. He was Program Cochairman for the 2000 International Symposium on Information Theory and Its Applications (ISITA) and Editor for the Proceedings of the 2006, 2003, and 1999 Symposiums on Applied Algebra, Algebraic Algorithms, and Error Correcting Codes (AAECC). 